\title{
EMG Analysis of Muscle Activity in Lower Limbs of Snowboarders
}

\author{
Jinho Back ${ }^{1}$, Wonil Son ${ }^{1}$, Jusung Lee ${ }^{1}$, Senghak Jo ${ }^{1}$, Jonggu $\mathrm{Yi}^{2}$ and \\ Siddhartha Bikram Panday ${ }^{1}$ \\ Kangwon National University, Department of Leisure Sports ${ }^{1}$, \\ BUZRUN Co., Ltd $^{2}$ \\ Gyodong 1,SamcheokSi, Kangwondo provice, Republic of Korea \\ ccdartha@gmail.com
}

\begin{abstract}
The objective of this research was to conduct comparative analysis of recruited muscles group of lower limbs and the muscle activity between alpine and boarder cross athlete in carving turn. Moreover the study aims to contribute snowboarders and winter sports instructors by providing appropriate training method and strength training methodology for performance enhancement. To achieve the purpose, one alpine and one boarder cross athlete from Korean National Team for 2018 Pyeongchang Winter Olympics were chosen. SEMG were placed on three muscle groups on the left side (Lt.) and right side (Rt.) muscles of lower limbs (vastus medialis oblique (VMO), vastus lateralis oblique (VLO), lateral gastrocnemius $(G L)$. The results obtained through the analysis are as follows. The results of comparative analysis in P1 showed the greatest recruitment of muscle activity in Rt.GL in alpine and Rt.VMO in boarder cross whereas, muscle activity in Lt.VLO were the lowest in both subjects. In P2, Lt.VLO in alpine and Rt.GL in boarder cross appeared the lowest whereas, Lt.VMO appeared the greatest in both of the subjects. In P3, Lt.VMO appeared greatest whereas, Rt.GL appeared the lowest in muscle activity in both subjects. In P4, Rt.GL appeared the lowest in both subjects. However, Rt.VMO showed the greater muscle activity in alpine and Lt.VMO showed greater muscle activity in boarder cross.
\end{abstract}

Keywords: EMG analysis, muscle activity, lower limbs

\section{Introduction}

Snowboarding emerged from an effort to enjoy surfing in winter. Snowboarding is an winter sporting event that gives pleasure through racing down the ski slopes using board. It has been an official sports in the Winter Olympics since the 1998 Winter Olympics Nagano, Japan. A total of four snowboarding event including slope style will be held at 2014 Winter Olympics Sochi, Russia

Among them, Alpine snowboarding involves strong and rough edging to carve. Moreover it is an event that involves extreme speed and thrill. Unlike freestyle snowboarding, Alpine snowboarding requires rigid boots that offers a much stiffer flex and allows for quicker edge change and more commanding edge pressuring. Therefore, it uses hard plastic injection molded type boots which look much like a ski boots. Bindings are also made of aluminum, plastic or carbon to resist the strong impact during snowboarding. The decks are also designed longer and stiffer than other freestyle decks, their breadth are narrower for high speed.

Boarder cross snowboarding is a mix between all mountain free riding, alpine racing and freestyle. It is a competition in which a group of four to six snowboarder racers start

This research project was supported by the 2012 Sports Promotion Fund of Seoul Olympic Sports Promotion Foundation from Ministry of Culture, Sports and Tourism | 
simultaneously atop a narrow inclined course and pass thru different obstacles to win. The course is narrower and contains a lot of physical contact. However, there are some limitations when pulling or push the opponent. Slowing down the opponent is also prohibited. Both alpine and freestyle snowboarders can participate in this event.

The technical phase of a snowboard turn starts from the traverse up and unweighting the pressure down through the fall line. Different stances and balance are used for various centering situation. Steering, rotation, pivoting are used to change the direction of the board; edging and turns can be achieved by combination of inclination and angulation. (Canadian Association of Snowboard Instructors, 2000; Hyun-dae Jo, 2011)

Carving turn is a technique in which riders initiate the turn by increasing the arc of the board to the surface and by leaving a precise slice carved arc in the snow. In carving turns, point of pressure that is conveyed to the board becomes slightly faster than in basic turns.

According to study conducted by Suk-Hwna Youn (2007) in Dynamic analysis and scientific education effect of Snowboard, while being in centered stance depending on the leg pressure applied to a snowboarder can initiate a front side or a back side turn. In addition, rapid edge transition while snowboarding can be experienced through applying opposite torque in the boots' front (toe) or the rear (heel) part which results in relatively faster edge change than during skiing.

The main purpose of carving turn is to prevent sliding and maintain speed through it. In order to maintain this speed and to control the snowboard muscular strength plays a vital role.

Christophe Delecluse (2001) concluded that front and rear legs don't share similar patterns of muscle activation while snowboarding with the up-unweighting technique in his study on muscle activity of knee extension (M. R.F and M. V.M) in different position between slalom and freestyle snowboarding. During frontside turn with slalom board and freestyle board activation of rectus femoris and vastus lateralis muscles was much higher in the rear leg. At backside turn with slalom board activation of rectus femoris and vastus lateralis muscles was lower in the rear leg compared to the front leg. Differences on a backside turn with freestyle board are less clear. There are also differences with muscle activation between the boards. Front leg has more activation while riding a slalom board, but with the rear leg this trend seems to be the opposite (Christophe Delecluse (2001.).

Despite EMG activity of lower limbs plays vital role during snowboard carving turn very few research has been conducted in this field. Moreover, this research aims to aid and develop the winter sports in Korea where researches in this field don't exist until now.

Based on this, the purpose of this study was to perform comparative analysis of recruited muscles groups of lower limbs in alpine and boarder cross athletes in carving turn and their muscle activity in different phases and also aims to contribute on providing appropriate training method and strength training methodology for performance enhancement to snowboarders and winter sports instructors.

\section{Method}

The study was conducted in order to perform comparative analysis of recruited muscles groups of lower limbs in alpine and boarder cross athletes in carving turn and their muscle activity in different phases. The experimental methods conducted in order to achieve the purpose of the study are presented as follows. 


\subsection{Subjects}

The subjects chosen for the experiment were one alpine and one boarder cross athlete from Korean National Team for 2018 Pyeongchang Winter Olympics. Physical characteristics of the subjects are presented in Table 1.

Table 1. Physical Characteristic of the Subjects

\begin{tabular}{|c|c|c|c|c|}
\hline Subjects & Height $(\mathbf{c m})$ & Weight $(\mathbf{k g})$ & Age & Experience (yrs) \\
\hline Alpine & 173 & 71 & 20 & 6 \\
\hline $\begin{array}{c}\text { Boarder } \\
\text { cross }\end{array}$ & 171 & 72 & 21 & 4 \\
\hline
\end{tabular}

\subsection{Experimental Procedure}

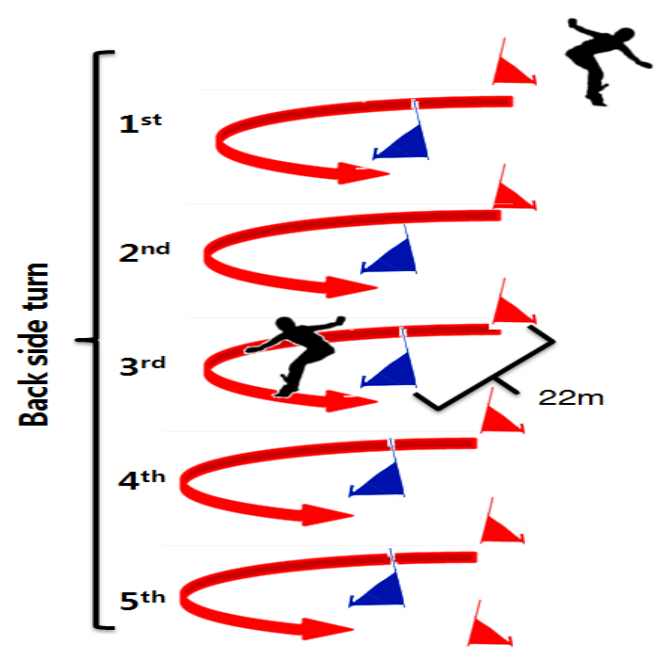

Figure 1. Experimental Slope

To investigate the muscle activity during the carving turn in snowboard, one alpine and one boarder cross athlete who are in Korean National Team Athlete for 2018 Pyeongchang Winter Olympics were chosen and SEMG were placed on three muscle groups on the left side (Lt.) and right side (Rt.) muscles of lower limbs (vastus medialis oblique (VMO), vastus lateralis oblique (VLO), lateral gastrocnemius (GL) and carving turns were conducted.

Before the experiment, the experimental procedures and possible risks were communicated verbally and in writing to all study participants, who then gave their informed written consent. The participants were allowed enough time to warm up and time to do two test run to familiarize with the slope conditions and to ensure actual competition condition. The test took place in the advanced ski slope at P ski resort, Kangwondo Korea. The experimental slope was $866 \mathrm{~m}$ in length at an altitude of $238.7 \mathrm{~m}$ with average pitch of 15.41 to maximum pitch of $36.0^{\circ}$ in the advanced course. The subjects performed two runs in a twenty four gates (Giant slalom Course) of which best turns were selected for analysis. The distance between each gate was about $22 \mathrm{~m}$. In addition, the phase from completion of front side turn to completion of back side turn were divided into four phases and the mean value of five consecutive back side turns were obtained. 


\subsection{Data Analysis}

EMG data obtained through the experiment were electrical raw data delivered directly from the active muscle groups through EMG module. Therefore, in order to achieve the purpose of the study integration of waveform and quantification was necessary to obtain precise data. Thus, in this study muscle activity patterns of three different major muscles on the left side (Lt.) and right side (Rt.) muscles of lower limbs Figure 2. [vastus medialis oblique (VMO), vastus lateralis oblique (VLO), lateral gastrocnemius(GL)] were analyzed through IEMG and full wave rectification was carried out in order to quantify the distortion and the signal characteristics. EMG signals were band-pass filtered at $20-500 \mathrm{~Hz}$ with the EMG full-wave rectified. Integrated EMG (IEMG) was derived by integrating the rectified signal with respect to specific time interval. In addition, the runs were divided into four phases out of which five continuous turns were selected for analysis and their mean values were calculated Figure1.

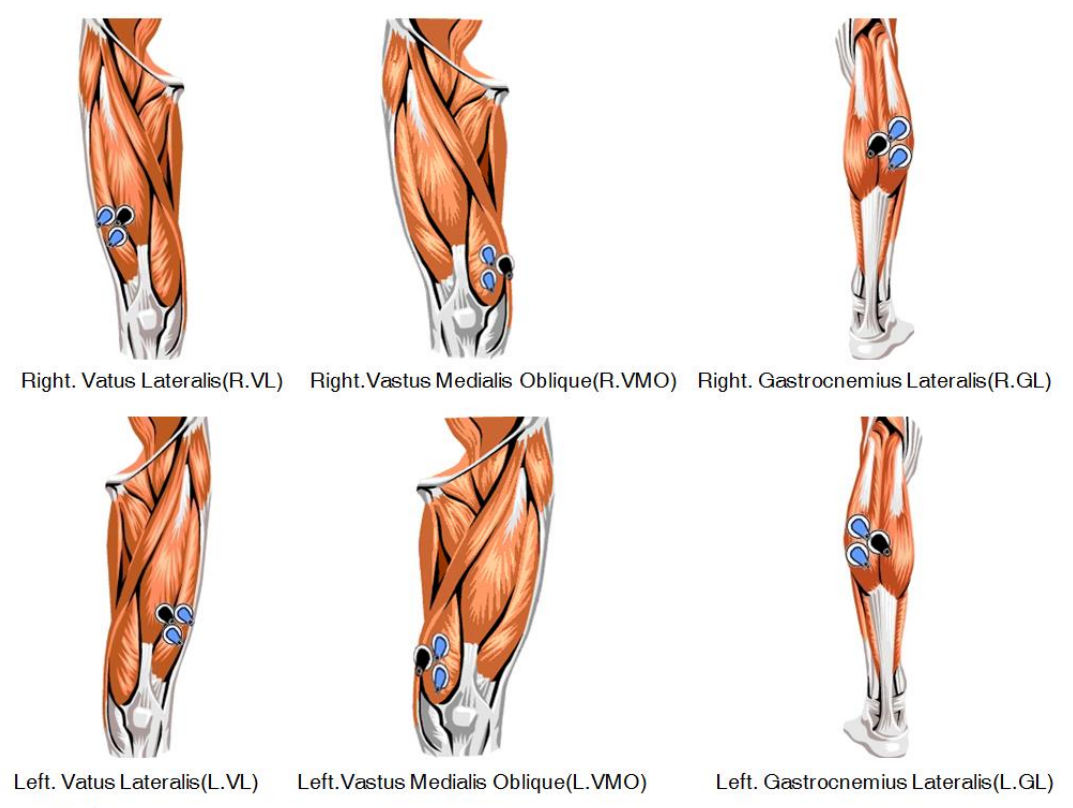

Figure 2. Placement of Electrodes in Lower Limbs for EMG Analysis

\subsection{Definition of Event and Phases}

The selection of events and phases of carving turn of Alpine snowboard athlete and Boarder cross athletes are as presented as follows <Figure 3.> .

\subsubsection{Definition of Event:}

E1: Starting point of Extension (up)

E2: Complete contact point of deck surface to the ground surface.

E3: Point of Complete flexion (down)

E4: Maximum angle of lean during back side turn

E5: Starting point of next extension (up) 


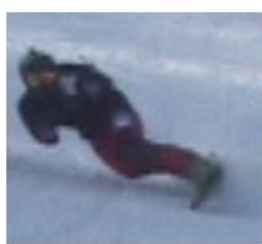

Event 1

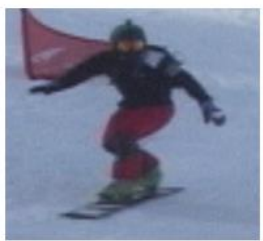

Event 2

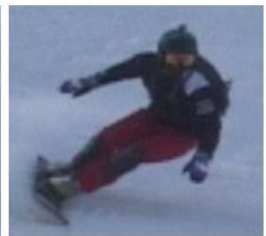

Event 3

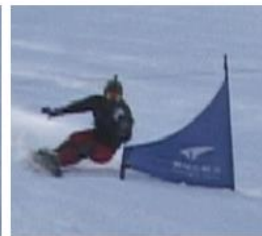

Event 4

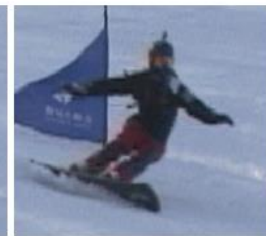

Event 5

Figure 3. Classification of Movement in Different Events

\title{
2.4.2. Definition of Phases
}

\begin{abstract}
P1 (Phase 1): From E1 to E2
P2 (Phase 2): From E2 to E3

P3 (Phase 3): From E3 to E4

P4 (Phase 4): From E4 to E5
\end{abstract}

\section{Results}

The study was conducted in order to perform comparative analysis of recruited muscles groups of lower limbs in alpine and boarder cross athletes' carving turn and their muscle activity in different phases. The results of the variation in patterns of different variables are presented as follows.

\subsection{Mean Value of Lower Limbs in Different Phases}

The mean value of recruited muscles of lower limbs of alpine and boarder cross snowboard athletes in different phases during carving turn are presented in Table 2 and Figure 4.

Table 2. Mean Value of Subjects' Lower Limbs in Different Phases [uV]

\begin{tabular}{|c|c|c|c|c|c|}
\hline & Muscles & P1 & $\mathbf{P 2}$ & P3 & P4 \\
\hline \multirow{6}{*}{ 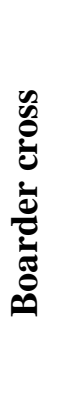 } & Rt.GL & $275.6 \pm 99.69$ & $141.4 \pm 15.32$ & $237.8 \pm 12.19$ & $286.4 \pm 121.77$ \\
\hline & Lt.GL & $180.8 \pm 66.62$ & $160.36 \pm 48.60$ & $249.2 \pm 112.99$ & $312.6 \pm 151.17$ \\
\hline & Rt.VMO & $397.4 \pm 126.88$ & $257 \pm 1532.57$ & $436 \pm 102.74$ & $478.8 \pm 75.28$ \\
\hline & Lt.VMO & $370 \pm 186.99$ & $349.6 \pm 42.92$ & $590.2 \pm 126.79$ & $714.8 \pm 250.55$ \\
\hline & Rt.VLO & $376.8 \pm 184.76$ & $266 \pm 34.77$ & $293.6 \pm 144.73$ & $366.6 \pm 153.49$ \\
\hline & Lt.VLO & $136.84 \pm 43.21$ & $167.6 \pm 27.46$ & $271.8 \pm 52.61$ & $297.8 \pm 88.38$ \\
\hline \multirow{6}{*}{ 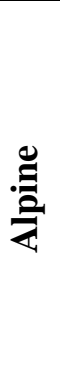 } & Rt.GL & $383.2 \pm 99.42$ & $251.06 \pm 202.81$ & $77.5 \pm 142.73$ & $117.38 \pm 74.25$ \\
\hline & Lt.GL & $174.6 \pm 69.73$ & $148.8 \pm 101.89$ & $148.98 \pm 41.63$ & $173.22 \pm 45.35$ \\
\hline & Rt.VMO & $269.0 \pm 98.97$ & $234.36 \pm 8.48$ & $120.2 \pm 65.46$ & $382.2 \pm 100.01$ \\
\hline & Lt.VMO & $369.6 \pm 76.39$ & $305 \pm 119.19$ & $257.2 \pm 79.08$ & $385 \pm 130.91$ \\
\hline & Rt.VLO & $198.74 \pm 64.80$ & $167.52 \pm 89.10$ & $95.04 \pm 29.86$ & $235 \pm 53.39$ \\
\hline & Lt.VLO & $137.6 \pm 18.69$ & $123.2 \pm 43.11$ & $123.3 \pm 47.90$ & $216 \pm 80.96$ \\
\hline
\end{tabular}


P1

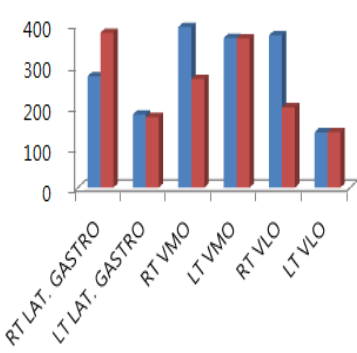

P3

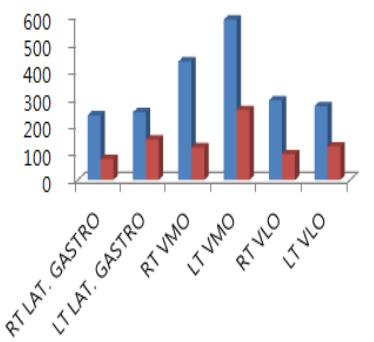

P2

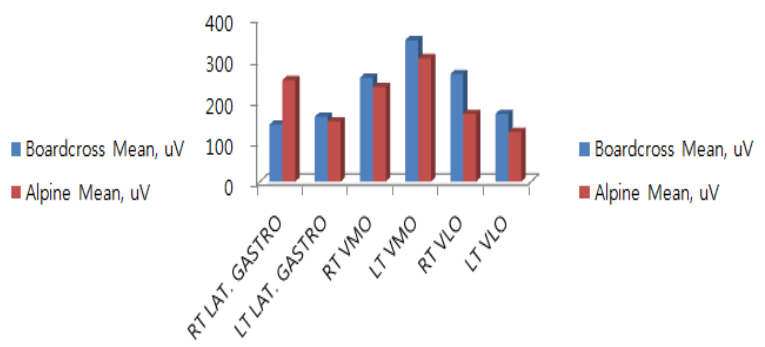

P4

- Alpine Mean, uV

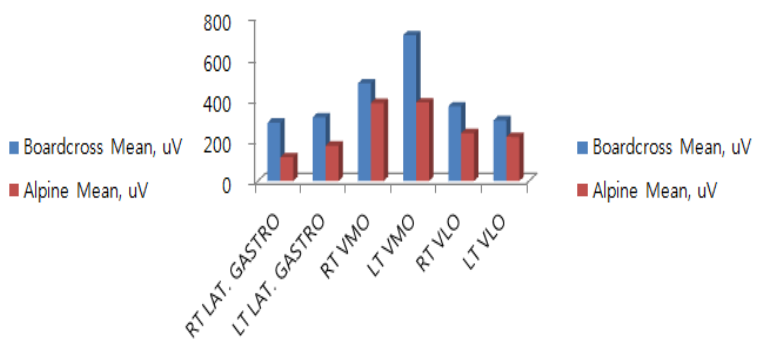

Figure 4. Mean Value of Subjects' Lower Limbs in Different Phases [uV]

The mean value of recruited muscles of lower limbs of boarder cross snowboard athlete during carving turn in P1, Rt.VMO $\left(397.4 \pm 126.88_{\mathrm{uV}}\right)$ showed the highest value whereas, Lt.VLO $\left(136.84 \pm 43.21_{\mathrm{uV}}\right)$ the lowest. In P2, Lt.VMO $\left(349.6 \pm 42.92_{\mathrm{uV}}\right)$ showed the highest value whereas, Rt.GL $\left(141.4 \pm 15.32_{\mathrm{uV}}\right)$ the lowest. In P3, Lt.VMO $\left(590.2 \pm 126.79_{\mathrm{uV}}\right)$ showed the highest value whereas, Rt.GL $\left(237.8 \pm 12.19_{\mathrm{uV}}\right)$ the lowest. In P4, Lt.VMO $\left(714.8 \pm 250.55_{\mathrm{uV}}\right)$ showed the highest. Whereas, Rt.GL $\left(286.4 \pm 121.77_{\mathrm{uV}}\right)$ the lowest.

The mean value of recruited muscles of lower limbs of alpine snowboard athlete during carving turn in P1, Rt.GL $(383.2 \pm 99.42$ uV) showed the highest value. Whereas, Lt.VLO $(137.6 \pm 18.69 \mathrm{uV})$ the lowest. In P2, Lt.VMO $(305 \pm 119.19 \mathrm{uV})$ showed the highest value. Whereas, Lt.VLO $\left(123.2 \pm 43.11_{\mathrm{uV}}\right)$ the lowest. In P3, Lt.VMO $\left(257.2 \pm 79.08 \mathrm{u}_{\mathrm{uV}}\right)$ showed the highest value. Whereas, Rt.GL (77.5 \pm 142.73 uV) the lowest In P4, Lt.VMO (385.0 $\pm 130.91 \mathrm{uV})$ showed the highest value. Whereas, Rt. GL $\left(117.38 \pm 74.25_{\mathrm{uv}}\right)$ the lowest.

Thus, both subjects in P2, P3 and P4 showed highest value in Lt.VMO. The results obtained are considered due to use of regular stance while snowboarding. In addition, Inclination arises in order to overcome the centrifugal force and the center of gravity shifted from the right to the left which is considered as a result to control speed while snowboarding. Based on Kyu-Kwon Cho (2007), in a study on waist and lower extremities muscle activities according to foot's positions during flexion/extension of legs VMO of lower extremities showed greater muscle activity when positioning them laterally. Contrarily, mean value of Rt.GL in P2, P3 and P4 appeared low. This is considered due to shift of center of gravity to the front side of the left leg from the right leg which decreased the muscle activity in it.

\subsection{Peak Value of Lower Limb's in Different Phases}

The peak value of recruited muscles of lower limbs of alpine and boarder cross snowboard athletes in different phase during carving turn are presented in Table 3 and Figure 5. 
Table 3. Peak Value of Subjects' Lower Limbs in Different Phases [uv]

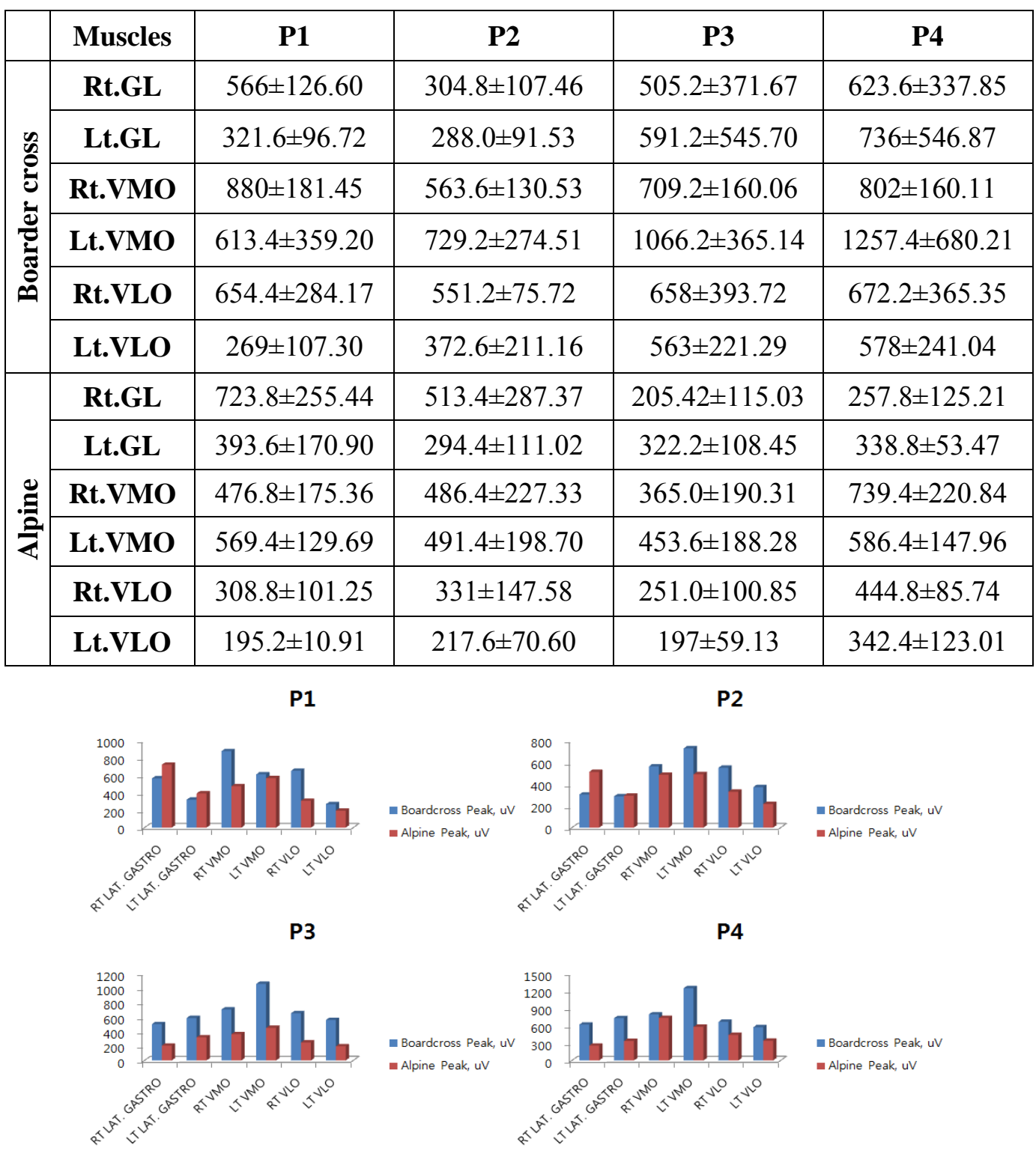

Figure 5. Peak Value of Subjects' Lower Limbs in Different Phases [uV]

The peak value of recruited muscles of lower limbs of boarder cross snowboard athlete during carving turn in P1, Rt.VMO $(880 \pm 181.45$ uV $)$ showed the highest value. Whereas, Lt.VLO $(269 \pm 107.30 \mathrm{uV})$ the lowest. In P2, Lt.VMO $(729.2 \pm 274.51 \mathrm{uV})$ showed the highest value. Whereas, Lt.GL $(288.0 \pm 91.53 \mathrm{uV})$ the lowest. In P3, Lt.VMO $(1066.2 \pm 365.14 \mathrm{uV})$ showed the highest value. Whereas, Rt.GL $\left(505.2 \pm 371.67_{\mathrm{uV}}\right)$ the lowest. In P4, Lt.VMO $\left(1257.4 \pm 680.21_{\mathrm{uV}}\right)$ showed the highest value. Whereas, Lt. VLO $\left(578 \pm 241.04_{\mathrm{uV}}\right)$ the lowest.

The peak value of recruited muscles of lower limbs of alpine snowboard athlete during carving turn in P1, Rt.GL ( $723.8 \pm 255.44$ uV) showed the highest value. Whereas, Lt.VLO $(195.2 \pm 10.91 \mathrm{uV})$ the lowest. In P2, Rt.GL $(513.4 \pm 287.37 \mathrm{uV})$ showed the highest value. Whereas, Lt.VLO $(217.6 \pm 70.60 \mathrm{uV})$ the lowest. In P3, Lt.VMO $(453.6 \pm 188.28 \mathrm{uV})$ showed the

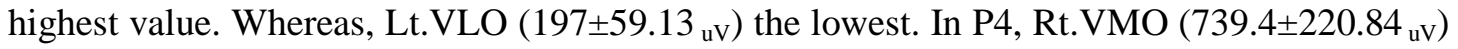


showed the highest value. Whereas, Rt.GL $(257.8 \pm 125.21$ uv $)$ the lowest. Also muscle activation measured with EMG reached near maximum levels in almost every turn.

Based on a study conducted by Berg \& Eiken (1999) while snowboarding with the upunweighting technique, the patterns of peak value between front and rear muscles of lower limbs didn't appear similar. Thus, Peak value of Lt.VMO showed highest in P2, P3 and P4 of boarder cross athlete. However, peak value Rt.GL generally showed highest in P1 and P2 of alpine snowboard athlete whereas, Lt.VLO mostly appeared low.

\subsection{Input\% of Muscles of Lower Limbs in Different Phase}

The Input\% value of recruited muscles of lower limbs of alpine and boarder cross snowboard athlete in different phase during carving turn are presented in Table 4 and Figure 6.

Table 4. Input\% of Muscles of Lower Limbs in Different Phase [\%]

\begin{tabular}{|c|c|c|c|c|c|}
\hline & Muscles & P1 & P2 & P3 & P4 \\
\hline \multirow{6}{*}{ 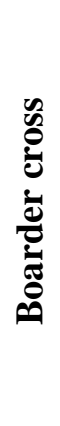 } & Rt.GL & $15.95 \pm 2.42$ & $10.66 \pm 1.53$ & $10.92 \pm 1.73$ & $11.72 \pm 3.20$ \\
\hline & Lt.GL & $10.51 \pm 1.29$ & $11.74 \pm 2.27$ & $11.64 \pm 1.53$ & $12.33 \pm 2.53$ \\
\hline & Rt.VMO & $23.70 \pm 5.57$ & $19.23 \pm 2.10$ & $21.39 \pm 2.15$ & $20.45 \pm 3.34$ \\
\hline & Lt.VMO & $20.62 \pm 4.38$ & $26.05 \pm 1.56$ & $29.11 \pm 2.73$ & $28.92 \pm 3.15$ \\
\hline & Rt.VLO & $21.00 \pm 2.35$ & $19.81 \pm 1.24$ & $13.40 \pm 2.56$ & $14.39 \pm 2.80$ \\
\hline & Lt.VLO & $8.22 \pm 1.82$ & $12.51 \pm 1.59$ & $13.53 \pm 1.66$ & $12.20 \pm 1.18$ \\
\hline \multirow{6}{*}{$\frac{\mathscr{E}}{\frac{2}{2}}$} & Rt.GL & $25.19 \pm 3.13$ & $18.43 \pm 4.73$ & $9.48 \pm 2.64$ & $7.36 \pm 2.64$ \\
\hline & Lt.GL & $11.09 \pm 1.82$ & $11.68 \pm 1.87$ & $18.37 \pm 1.88$ & $11.55 \pm 1.66$ \\
\hline & Rt.VMO & $17.30 \pm 2.72$ & $18.35 \pm 6.20$ & $14.22 \pm 2.98$ & $25.64 \pm 4.46$ \\
\hline & Lt.VMO & $24.34 \pm 1.98$ & $26.51 \pm 2.71$ & $31.29 \pm 1.32$ & $25.22 \pm 3.26$ \\
\hline & Rt.VLO & $12.93 \pm 1.98$ & $14.01 \pm 3.45$ & $11.74 \pm 1.31$ & $16.22 \pm 3.40$ \\
\hline & Lt.VLO & $9.15 \pm 0.64$ & $11.01 \pm 1.51$ & $14.91 \pm 1.36$ & $14.01 \pm 1.47$ \\
\hline
\end{tabular}

P1

P2

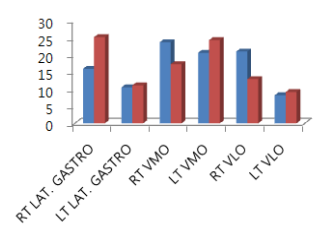

P3

In Boardcross Input, \% = Alpine Input, \%

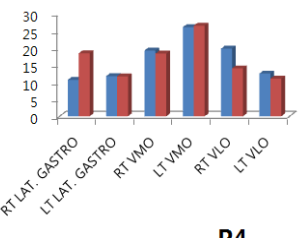

n Boardcross Input, $\%$

- Alpine Input, \%

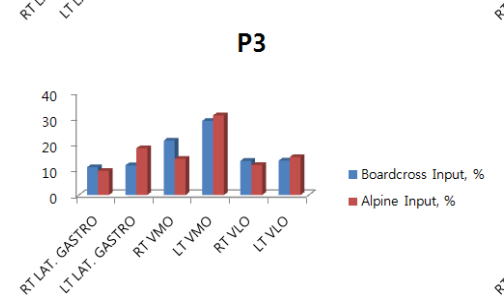

P4

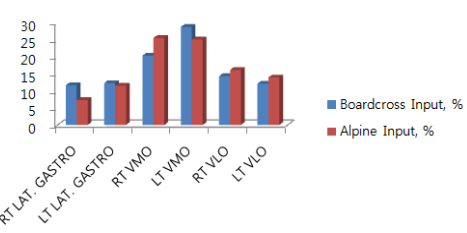

Figure 6. Input\% of Muscles of Lower Limbs in Different Phase [\%] 
The Input\% value of recruited muscles of lower limbs of boarder cross snowboard athlete during carving turn in P1 was Rt. VMO (23.70 \pm 5.57$)$ showed the highest value. Whereas, Lt.VLO $(8.22 \pm 1.82)$ the lowest. In P2, Lt.VMO $(26.05 \pm 1.56)$ showed the highest value. Whereas, Rt.GL $(10.66 \pm 1.53)$ the lowest. In P3 Lt.VMO $(29.11 \pm 2.73)$ showed the highest value. Whereas, Rt.GL $(10.92 \pm 1.73)$ the lowest. In P4 Lt.VMO (28.92 \pm 3.15$)$ showed the highest value. Whereas, Rt.GL (11.72 \pm 3.20$)$ the lowest.

The Input\% value of recruited muscles of lower limbs of alpine snowboard athlete during carving turn in P1, Rt.GL (25.19 \pm 3.13$)$ showed the highest value. Whereas, Lt.VLO $(9.15 \pm 0.64)$ the lowest. In P2, Lt.VMO $(26.51 \pm 2.71)$ showed the highest value. Whereas, Lt.VLO (11.01 \pm 1.51$)$ the lowest. In P3, Lt.VMO (31.29 \pm 1.32$)$ showed the highest value. Whereas, Rt.GL (9.48 \pm 2.64$)$ the lowest. In P4, Rt.VMO (25.64 \pm 4.46$)$ showed the highest value. Whereas, Rt.GL (7.36 \pm 2.64$)$ the lowest.

During front side turn with slalom board and freestyle board activation of rectus femoris and vastus lateralis muscles is higher in the rear leg. Whereas during backside turn with slalom board, activation of rectus femoris and vastus lateralis muscles is lower in the rear leg compared to the front leg. Differences on a backside turn with freestyle board are less clear. There are also differences with muscle activation between the boards. Front leg has more activation while riding a slalom board, but with the rear leg trend seems to be the opposite (Delecluse et al., 2001.).

In boarder cross athlete, Lt.VMO showed greater muscle activity in P2, P3 and P4 whereas, Rt.GL the lowest. In alpine snowboard athlete, Lt.VMO showed greater muscle activity in P2 and P3 and greater muscle activity was observed in Lt.VLO in P1 and P2. However, in P4 low muscle activity appeared in Rt.GL. In general, Rt.VMO showed greater muscle activity in P1 in boarder cross athlete whereas, L.VLO appeared the lowest. However, R.GL showed greater muscle activity in P1 in alpine snowboard athlete whereas, Lt.VLO appeared the lowest. Even though, similar section in carving turn of both subjects were analyzed the differences in result obtained is considered due to the properties of equipments used, angle of binding, stance width and differences in their riding techniques.

\section{Conclusion and Discussion}

The purpose of this study was to perform comparative analysis of recruited muscles groups of lower limbs in alpine and boarder cross athletes in carving turn and their muscle activity in different phases. Accordingly, this study aims to contribute on providing appropriate training method and strength training methodology for performance enhancement to snowboarders and winter sports instructors. The conclusion and discussion of the comparative analysis are presented as follows.

1. In both subjects mean value of Lt.VMO showed the highest value in P2, P3 and P4 which is considered due to use of regular stance while snowboarding. In addition, Inclination arises in order to overcome the centrifugal force and the center of gravity shifts from the right to the left which is considered as a result to control speed while snowboarding. Contrarily, Mean value of Rt.GL in P2, P3 and P4 appeared low. This was due to shift of center of gravity to the front side of the left leg. Contrarily, Mean value of Rt.GL in P2, P3 and P4 appeared low. This is considered due to shift of center of gravity to the front side of the left leg from the right leg which decreased the muscle activity in it.

2. Peak value of Lt.VMO appeared highest in P2, P3 and P4 of boarder cross athlete. Contrarily, peak value Rt.GL generally appeared highest in P1 and P2 of alpine snowboard athlete whereas, Lt.VLO mostly appeared low. The patterns of peak value between front and rear muscles of lower limbs didn't appear similar while snowboarding with the up- 
unweighting technique. These result appeared similar to the result as of the preceding research. In conclusion, it is considered that the trembling of recruited muscles while snowboarding can be minimized through training and speedy turns can be controlled safely.

3. The Input\% value of muscle activity in lower limbs in different phases in boarder cross athlete, Lt.VMO showed greater muscle activity in P2, P3 and P4 whereas, Rt.GL the lowest. In alpine snowboard athlete, Lt.VMO showed greater muscle activity in P2 and P3 whereas, low muscle activity appeared in Lt.VLO in P1, P2 and Rt.GL in P3, P4. In general, in boarder cross athlete Rt.VMO showed greater muscle activity in P1 whereas, Lt.VLO appeared the lowest. However, in alpine snowboard athlete Rt.GL showed greater muscle activity in P1 whereas, Lt.VLO appeared the lowest. After edge change during back side turn inclination arises in order to overcome the centrifugal force and the center of gravity shifts from the right to the left which is considered as a result to control speed where Lt.VMO showed greater muscle activity than Rt.GL which generally showed lower muscle activity.

Even though, similar section in carving turn were analyzed the differences in results are considered due to the influence of different factors such as the properties of equipment, angle of binding, stance width, differences in their riding techniques which can correlate and affect the muscle activity during carving turn. Therefore, further research in these areas is needed. Accordingly, the efficiency of recruited muscles while snowboarding can be improved through different strength training and balance training program. Moreover, this study can enhance the performance of the snowboarders and can aid by providing appropriate snowboarding as well as strength training methods to winter sports instructors.

\section{References}

[1] A. Mirzal, "Stability Analysis and Compensation of Time Delays in Analog Control Systems", IJ. Control and Automation, SERSC, vol. 5, (2012), pp. 1-18.

[2] A. Bakken, T. Bere, R. Bahr, E. Kristianslund and L. Nordsletten, "Mechanisms of injuries in World Cup Snowboard Cross: a systematic video analysis of 19 cases", British Journal of Sports Medicine, (2011), pp. 1315-1322.

[3] B.-J. Kang and Y. Seock Lee, "A Study on the Measuring EMG Signal Using Two-Dimensional images of the Fatigue analysis", Dept. of Digital broadcasting and Electronics Eng., Chungwoon University, Journal of the Korea Academia-Industrial cooperation Society, (2009), pp. 1010-1013.

[4] Canadian Association of Snowboard Instructors, Canadian Association of Snowboard Instructors guide book, www.casi-acms.com, (2000).

[5] K.-K. Cho, Y.-S. Kim, S.-M. Yang and W.-W. Son, "A Study on waist and lower extremities muscle activities according to foot's positions during flexion/extension of legs", The Korea Journal of Sports science, vol. 6 , no. 3, (2007), pp. 737-747.

[6] C. Delecluse, T. Coeckelberghs and R. Vranken, "Effects of the different position of the feet in slalom and freestyle snowboarding on muscle activity of knee extensors", International Symposium on Biomechanics in Sports, (2001), pp. 62-65.

[7] D.-Y. Kim and J.-H. Song, "Snowboarding with Professionals", Daesun publishing, Korea, ISBN: 89-8894441-0 93690, (2004).

[8] R. A. Hintermeister, G. W. Lange, D. D. O’Connor, C. J. Dillman and J. R. Steadman, "Muscle activity of slalom and giant-slalom skiing", Official Journal of the American College of Sports Medicine, (1994), pp. 315-322.

[9] J.-H. Park and C.-S. Jeong, "Real-time Signal Light Detection”, IJ. Signal Processing, Image Processing and Pattern Recognition, SERSC, vol. 2, (2009), pp. 1-10.

[10] J. Hyun-Dae, "The Kinematic Comparative Analysis of the Snowboard turn", Postgraduate thesis, Graduate School of Korea National University of Education, Korea, (2011).

[11] J.-S. Park, J.-H. Yoon and C. Kim, "Stable 2D Feature Tracking for Long Video Sequences", IJ. Signal Processing, Image Processing and Pattern Recognition, SERSC, vol. 1, (2008), pp. 39-46.

[12] M. S. Kocher, M. M. Dupré and J. A. Feagin, Jr, "Shoulder injuries from alpine skiing and snowboarding. Aetiology", treatment and prevention. Sports Medicine, (1998), pp. 201-211.

[13] Korea Ski Association Snowboard Committee, Korea Ski Association Snowboard Instructor Text Book (2004), http://www.ksia.co.kr/, vol. 2, pp. 50. 
[14] L. Chong Hoon, "Biomechanical Analysis of the Physical Effects of Riding the X-lider", IJ. Bio-Science and Bio-Technology, SERSC, vol. 3, (2011), pp. 69-78.

[15] N. Joung Kwak and T.-S. Song, "Joint Tracking and Transmission System for Simulating Motion of the Human Body on Android Smart Phone", IJ. Control and Automation, SERSC, vol. 4, (2011), pp. 81-90.

[16] R. B. Abu-laban MD, "Snowboarding injuries: an analysis and comparison with alpine skiing injuries", CAN MED ASSOC J, (1991), pp. 145.

[17] A. Satya Kalyan, K. N. Rao Divakar T and A. Mani Chakravarthi, "Vehicle Velocity Prediction \& Estimation in 2d Video for Night Condition", IJ Signal Processing, Image Processing and Pattern Recognition, SERSC, vol. 4, (2011), pp. 19-30.

[18] S. Jo Han and S.-U. Kim, "Comparison of Peak EMG Amplitude on Low Back Muscles according to Asymmetric Load Center of Gravity and Trunk Lateral Bending while Lifting”, Department of Industrial Engineering, Dankook University, Journal of the Korea Academia-Industrial cooperation Society, http://dx.doi.org/10.5762/KAIS.2012.13.10.4629, vol. 13, (2012), pp. 46294635.

[19] S.-H. Cho and S.-G. Kim, "The Effect of Depending on Variations of Speed in Backward Walking on Lower Extremities Muscle", Dept. of Rehabilitation Science, Graduate School of Daegu University, Journal of the Korea Academia-Industrial cooperation Society, http://dx.doi.org/10.5762/KAIS.2012.13.5.2199, vol. 13, (2012), pp. 2199-2205.

[20] Y. Suk-Hwna, "Dynamic analysis and scientific education effect of Snowboard", Postgraduate thesis, Department of Physics Education, the graduate School of Education, Hankuk University of Foreign Studies, Korea, (2007).

\section{Authors}

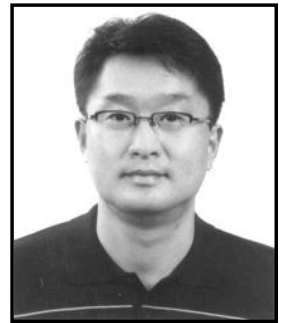

Jin ho Back, is a professor at Department of Leisure Sports, Kangwon National University Samcheok campus. He received his Ph.D. degree from Sungkyunkwan University, Korea in 1997. He was a senior researcher at Korea institute of Sports science from 1997 to 2008. Currently, He is an executive director as well as editor of Korean Society of Sports Biomechanics (KSSB). His research interests include Sports Biomechanics and Motion Analysis.

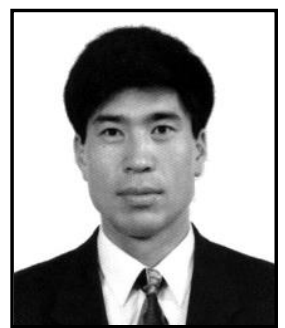

Won Il Son, received his B.Ed, M.Ed in Physical Education and Ph.D. degree from Hanyang University, Korea in 1999. Currently, Prof. Son is a faculty member with the Leisure Sports Department, Kangwon National University Samcheok Campus in Korea. Prof. Son is a member of Korean Alliance for Health, Physical Education, Recreation and Dance (KAHPERD). His research interest includes Physical Evaluation and Measurement.

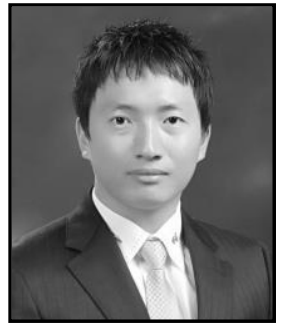

Jusung Lee, has received his B.Ed and M.Ed in Physical Education from Kangwon National University, Korea in 2009 and 2013 respectively. Currently, he is a researcher in a project for Development of Measurement and Evaluation in Ski and Snowboard Based on Sports Science which is supported by Ministry of Culture, Sports and Tourism. His field of interest includes Sports Biomechanics, EMG Analysis, Motion Analysis, Motor Control and Disable Sports. 


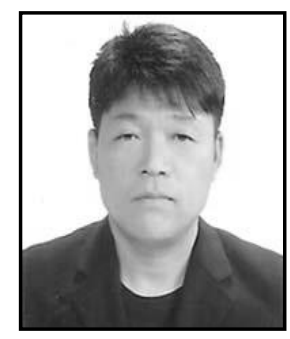

Senghak Jo, he is a graduate student majoring Physical Education in Kangwon National University, Korea. Mr. Jo is a vice president of Kangwondo Football Association for disabled as well as head coach of Korean Football Association of Mentally Disabled.

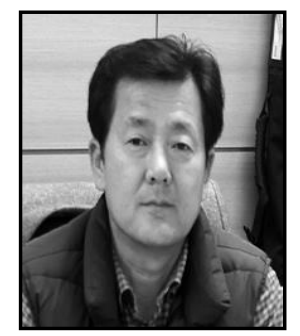

Jonggu Yi, is CEO of Buzrun Co. Ltd which is and He was the fifth consecutive president of Korean Snowboard Association from 1993 to 1995. Since 1994, he has been producing snowboard in Korea and has registered more than 10 patents in the related field. Moreover, He has been working to develop the snowboarding field in Korea by organizing different Buzrun Pro and Amateur Snowboard Competition.

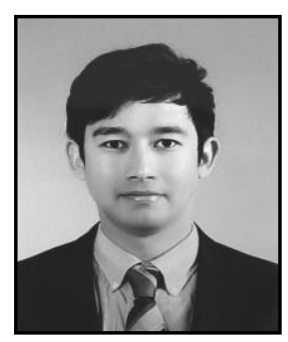

Siddhartha Bikram Panday, he is currently a graduate student majoring Physical Education at Kangwon National University, Korea. Currently, $\mathrm{He}$ is a researcher in a project for Development of Measurement and Evaluation in Ski and Snowboard Based on Sports Science which is supported by Ministry of Culture, Sports and Tourism. His field of interest includes Sports Biomechanics, EMG Analysis, Motion Analysis, Physical Evaluation and Measurement and Taekwondo. 